\title{
The dog as an animal model for bladder and urethral urothelial carcinoma: Comparative epidemiology and histology
}

\author{
SIMONE DE BROT ${ }^{1}$, BRIAN D. ROBINSON ${ }^{2}$, TIM SCASE $^{3}$, LLORENÇ GRAU-ROMA ${ }^{1}$, \\ ELEANOR WILKINSON ${ }^{1}$, STEPHEN A. BOORJIAN $^{4}$, DAVID GARDNER $^{1}$ and NIGEL P. MONGAN ${ }^{1,2}$ \\ ${ }^{1}$ School of Veterinary Medicine and Science, University of Nottingham, Sutton Bonington, LE12 5RD, UK; \\ ${ }^{2}$ Department of Pathology, Weill Cornell Medical College, New York, NY 10065, USA; ${ }^{3}$ Bridge Pathology \\ Ltd., Bristol, BS7 0BJ, UK; ${ }^{4}$ Department of Urology, Mayo Clinic, Rochester, MN 55905, USA
}

Received October 9, 2017; Accepted February 21, 2018

DOI: $10.3892 / 01.2018 .8837$

\begin{abstract}
Despite the recent approval of several novel agents for patients with metastatic urothelial carcinoma (UC), survival in this setting remains poor. As such, continued investigation into novel therapeutic options remains warranted. Pre-clinical development of novel treatments requires an animal model that accurately simulates the disease in humans. The aim of the present study was to evaluate the dog as an animal model for human UC. A total of 260 cases of spontaneous, untreated canine primary urethral and urinary bladder UC, were epidemiologically and histologically assessed and classified based on the current 2016 World Health Organization (WHO) tumor classification system. Canine data was compared with human data available from scientific literature. The mean age of dogs diagnosed with UC was 10.22 years (range, $4-15$ years), which is equivalent to 60-70 human years. The results revealed a high association between UC diagnosis with the female sex [odds ratio (OR) 3.51; 95\% confidence interval (CI) 2.57-4.79; $\mathrm{P}<0.001$ ], surgical neutering (OR 4.57; 95\% CI 1.87-11.12; $\mathrm{P}<0.001)$ and breed (OR 15.11 for Scottish terriers; 95\% CI 8.99-25.41; $\mathrm{P}<0.001)$. Based on the 2016 WHO tumor $(\mathrm{T})$, node and metastasis staging system, the primary tumors were characterized as T1 (38\%), T2a (28\%), T2b (13\%) and T3 (22\%). Non-papillary, flat subgross tumor growth was strongly associated with muscle invasion (OR 31.00; $\mathrm{P}<0.001)$. Irrespective of subgross growth pattern, all assessable tumors were invading beyond the basement membrane compatible with infiltrating UC. Conventional, not further classifiable infiltrating UC was the most common type of tumor (90\%), followed by UC with divergent, squamous and/or glandular
\end{abstract}

Correspondence to: Dr Simone de Brot, School of Veterinary Medicine and Science, University of Nottingham, Sutton Bonington Campus, College Road, Sutton Bonington, LE12 5RD, United Kingdom

E-mail: simone.debrot@nottingham.ac.uk

Key words: urothelial carcinoma, bladder, urethra, dog, animal model differentiation (6\%). Seven out of the $260(2.8 \%)$ cases were classified as non-urothelial based on their histological morphology. These cases included 5 (2\%) squamous cell carcinomas, $1(0.4 \%)$ adenocarcinoma and $1(0.4 \%)$ neuroendocrine tumor. The 2 most striking common features of canine and human UC included high sex predilection and histological tumor appearance. The results support the suitability of the dog as an animal model for UC and confirm that dogs also spontaneously develop rare UC subtypes and bladder tumors, including plasmacytoid UC and neuroendocrine tumor, which are herein described for the first time in a non-experimental animal species.

\section{Introduction}

Bladder cancer (BC) is the ninth most common malignancy worldwide and the most common cancer involving the urinary system. BC comprises approximately $2 \%$ of all cancers, with about 330,000 new BC cases each year globally $(1,2)$. The highest incidence rates are observed in highly developed countries, where BC is the fourth and 14th most common cancer in men and women, respectively (2). Despite ongoing effort to improve therapeutic options, there is still no curative therapy available for metastatic disease. The pre-clinical development of new treatments requires an animal model that accurately simulates the disease in people. Rodent animal models with experimentally induced bladder cancer have been shown to be valuable, but their translational relevance has been limited (3-5). Therefore, well characterized spontaneous bladder cancers in companion animals could represent a useful tool to advance the development of improved diagnostics and therapeutics. Indeed dogs develop spontaneous $\mathrm{BC}$, more specifically urothelial carcinoma (transitional cell carcinoma), with striking clinical, histopathological and molecular similarities compared to humans (6). Furthermore, companion animals and their owners share the environment, including exposure to carcinogenic components. In addition, other advantages of using spontaneous urinary bladder cancer in pet dogs as an animal model for human UC are: i) reduced use of research animals, ii) the shorter lifespan of dogs, relative to people, makes time-efficient clinical studies possible and iii) dogs show a similar response to therapy used 
to treat people (7-11). Yet one limitation to the use of the dog in preclinical studies of $\mathrm{BC}$ has been access to robust clinical and pathologic datasets. For this reason, the present study, which is one of the largest yet undertaken comparative studies, aims to evaluate the dog as animal model for human urothelial carcinoma by comparing the epidemiology and histology of $\mathrm{n}=260$ canine urinary bladder and urethral urothelial carcinoma to the data available in literature regarding their human counterpart.

\section{Materials and methods}

The present study was reviewed and approved by the University of Nottingham's ethical review committee. Informed written consent was obtained from the dog owners upon sample submission. All patient data has been anonymized. The present retrospective study includes archived formalin-fixed paraffin-embedded (FFPE) tissues from 260 dogs with primary urethral $(n=61)$ or urinary bladder $(n=199)$ carcinomas. All samples were taken from pet dogs living in the United Kingdom (UK) and were originally submitted for diagnostic purposes. Two hundred fifty-nine cases were provided by a private diagnostic pathology laboratory (Bridge Pathology Ltd., Bristol, UK) which received the tissue samples between October 2008 and April 2015. One additional case of canine urinary bladder carcinoma which was submitted to the University Nottingham Veterinary Pathology Service in May 2016 was also included. Non-invasive urothelial lesions, including papillomas, were excluded from the study due to the low number of cases in the present dog population. Tissue of canine in situ UC was not available. From the originally retrieved $n=265$ cases of UC, two cases were excluded due to poor tissue section quality, another two cases due to lack of convincing neoplastic features, and one case due to lack of information about dog breed and age. The following case information was available: Age at the time of first tumor diagnosis, sex, neutering status, and dog breed. All canine cases $(n=93,862)$ submitted to the same laboratory within the same time period were used as control population. These control dogs were diagnosed with a wide range of neoplastic and non-neoplastic diseases in various organs and tissues, excluding carcinoma of the lower urinary tract. Haematoxylin and eosin stained tissue sections of all 260 cases were available which were digitalized (scanner 3DHISTECH Pannoramic 250 Flash III) and assessed using the software 3DHISTECH Case Viewer by a board-certified veterinary pathologist ( $\mathrm{SdB}$ ), with support of a certified human uropathologist (BR) and a second veterinary pathologist (LGR). All cases were histologically assessed for tumor stage and histological subtype, based on the World Health Organization (WHO) tumor classification system 2016 (12). Subgross tumor growth patterns were evaluated histologically at a low magnification (1 and 2x). Epidemiological and pathological canine data was compared with data available from scientific human literature which was available on PubMed (https://www.ncbi.nlm.nih. gov/pubmed/) in April 2017. Cancer incidence was calculated in percentage $(\%)$ for each dog breed ( $n=60$ different breeds). $100 \%$ cancer incidence was defined as the total number of dogs of the same breed in the control population. Breeds with incidence rates above average $(>0.823 \%)$ and with a minimum of $n=5$ cancer cases were defined as risk breeds. Three different breeds (Scottish terrier, Shetland sheepdog and West Highland White terrier) fulfilled these criteria and were grouped together as 'risk breeds'. 'Non-risk dog breeds' were defined as all dog breeds, including cross breeds, excluding Scottish terriers, Shetland Sheepdogs, and West Highland White terriers. Odds ratios were calculated in order to quantify the risk of dog breed, sex, neutering status with the development of cancer, and in order to quantify the risk of non-papillary tumor growth with muscle-invasive tumor growth. An independent t-test was performed to compare the mean age of risk breeds vs. non-risk breeds, of dogs with muscle-invasive vs. non muscle-invasive tumor growth, and of dogs with papillary vs. non-papillary tumor growth, respectively. Chi-square test was used to test for associations between tumor incidence and dog breed, sex, and neutering status, and to analyze the association of tumor invasive growth with tumor growth pattern, dog breed, and sex. Binary logistic regression analysis was performed in order to identify and weigh risk factors, including dog breed, sex, and neutering. $\mathrm{P}<0.05$ was considered to indicate a statistically significant difference. Statistical analyses were performed using SPSS v.22.0 (IBM Corp., Armonk, NY, USA).

\section{Results}

A total of 260 dogs with spontaneous urinary bladder (199/260, $77 \%)$ and primary urethral $(61 / 260,23 \%)$ urothelial carcinoma (UC) were included in this study. Tables I and II summarize the main canine epidemiological findings and compare them with the corresponding human data available in the literature.

The mean age of dogs diagnosed with UC was older compared to dogs without UC (10.21 [+/-2.0, range 4-15] years vs. 7.1 [+/-3.6] (mean +/-SD, $\mathrm{P}<0.001)$. Dog breeds with only 1 case of UC were excluded from any further analysis (leaving 66,317 cases; $222(0.3 \%)$ with UC). In univariate analyses [odds ratio ( $95 \%$ confidence interval), $\mathrm{P}<0.001$ ], female sex [OR, 3.51 (2.57-4.79)], neutering [OR, 4.57 (1.87-11.12)] and breed (OR 15.11 [8.97-25.41] for Scottish terriers, OR 6.82 [3.01-15.49] for Shetland sheepdogs, and OR 2.79 [1.80-4.35] for West Highland white terriers) were identified as significant predictors of UC (Table I). All remained significant $(\mathrm{P}<0.001)$ in multivariate analyses (Table $\mathrm{I})$. In addition, when the three top risk breed dogs were grouped together, they were found to be diagnosed with UC at a significantly younger age [mean 112.14 months (9.35 years), SD 19.16] compared to the group containing 'non-risk dog breeds' [mean 124.69 months (10.39 years), SD 24.74] $(\mathrm{P}<0.001)$. This effect was most evident for female neutered Scottish terriers (Fig. 1). 'Non-risk dog breeds' were defined as all dog breeds, including cross breeds, excluding Scottish terriers, Shetland Sheepdogs, and West Highland White terriers.

All cases were histologically assessed for tumor stage and histological subtype, based on the World Health Organization (WHO) tumor classification system $2016(12,13)$. Tumor location, growth pattern and main histological features of the studied dogs are summarized in Table III together with the equivalent information extracted from the human literature.

Gross tumor location was available for 29/199 (15\%) bladder and 10/61 (16\%) urethral UC cases. The most common location within the bladder was the neck $(14 / 29$, $48 \%)$, followed by the trigone $(9 / 29,28 \%)$, cranial $(3 / 2910 \%)$, 
Table I. Risk factors for urothelial carcinoma in the studied dog population.

\begin{tabular}{|c|c|c|c|c|c|c|c|c|}
\hline Variables & $\begin{array}{l}\text { Absolute } \\
\text { nos. }\end{array}$ & $\begin{array}{c}\text { Prevalence } \\
(\%)\end{array}$ & $\begin{array}{l}\text { Univariate } \\
\text { OR }\end{array}$ & $95 \% \mathrm{CI}$ & P-value & $\begin{array}{l}\text { Multivariate } \\
\text { OR }\end{array}$ & $95 \% \mathrm{CI}$ & P-value \\
\hline Female sex & & & 3.51 & $2.57-4.79$ & $<0.001$ & 2.92 & $2.17-3.91$ & $<0.001$ \\
\hline With UC & 170 & 77 & & & & & & \\
\hline Without UC & 31,243 & 48 & & & & & & \\
\hline Neutering & & & 4.57 & $1.87-11.12$ & $<0.001$ & 3.75 & $1.85-7.60$ & $<0.001$ \\
\hline With UC & 166 & 75 & & & & & & \\
\hline Without UC & 37,054 & $88^{\mathrm{a}}$ & & & & & & \\
\hline \multicolumn{9}{|l|}{ Breed } \\
\hline Scottish terrier & & & 15.11 & $8.99-25.41$ & $<0.001$ & 19.51 & $11.48-33.16$ & $<0.001$ \\
\hline With UC & 16 & 7 & & & & & & \\
\hline Without UC & 338 & 0.5 & & & & & & \\
\hline Shetland sheepdog & & & 6.82 & $3.01-15.49$ & $<0.001$ & 12.33 & $5.35-28.40$ & $<0.001$ \\
\hline With UC & 6 & 3 & & & & & & \\
\hline Without UC & 268 & 0.4 & & & & & & \\
\hline $\begin{array}{l}\text { West Highland } \\
\text { white terrier }\end{array}$ & & & 2.79 & $1.80-4.35$ & $<0.001$ & 3.30 & $2.03-5.36$ & $<0.001$ \\
\hline With UC & 22 & 10 & & & & & & \\
\hline Without UC & 2,504 & 4 & & & & & & \\
\hline
\end{tabular}

Female sex, neutering and certain dog breeds were identified as risk factors by univariate and multivariate regression analysis. ${ }^{\mathrm{a} N o t e}$ that neutering status was unknown in 23,997 (36\%) of cases. CI, Confidence interval; OR, odds ratio; UC, urothelial carcinoma.

ventral $(1 / 29,4 \%)$ and dorsal $(1 / 29,4 \%)$ bladder. In $2 / 29$ cases (7\%), tumor growth was present in both bladder neck and cranial areas. The location of urethral UC was more common in the distal $(8 / 10,80 \%)$ than in the proximal $(2 / 10,20 \%)$ urethra.

The subgross tumor growth pattern could be assessed in 66/199 (33\%) bladder UC cases, which were provided as full transmural tissue sections. Two different types of subgross growth appearance were recognized, papillary and non-papillary (also referred to as flat). Papillary UC (Fig. 2A) were equally as common $(33 / 66,50 \%)$ as flat (Fig. 2B) $(33 / 66$, $50 \%$ ) tumors, with identical female: Male ratio of 1: 1.57 in both growth patterns $(21 / 33,67 \%$ females, $12 / 33,33 \%$ males). Irrespective of growth pattern, all UC were invading beyhond the basement membrane, compatible with infiltrating UC based on the WHO 2016 tumor classification.

When applying the tumor, node and metastasis (TNM) staging system provided by the 2016 WHO classification system (12), tumor characterization was limited to the assessment of the primary tumor. Information about the involvement of lymph nodes and presence of distant metastatic spread was not available, which prevented the performance of an overall tumor staging. The extent of tumor infiltration into the bladder wall could be assessed in 64/199 (32\%) bladder UC cases which were available as transmural tissue sections. Thirty-eight \% (24/64) of cases were limited to the lamina propria mucosae (T1), 28\% (18/64) invaded the superficial muscularis propria (T2a), and $13 \%(8 / 64)$ the deep muscularis propria (T2b). A substantial number $(14 / 64,22 \%)$ of UC cases invaded perivesicular tissue (T3). Muscle-invasive growth could be assessed in 76/199 (38\%)
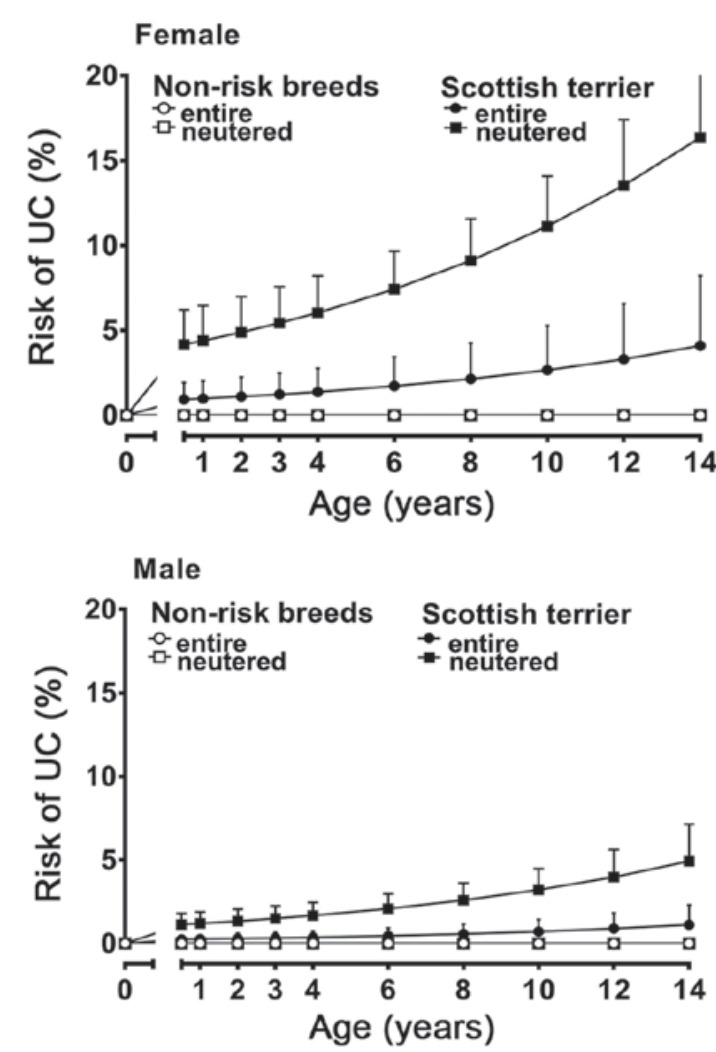

Figure 1. Effect of sex, neutering, age and dog breed on cancer incidence. The risk of UC in Scottish terriers increased with age. This effect was the greatest in female neutered dogs. Non-risk breeds were defined as all dog breeds including cross breeds, excluding Scottish terriers, Shetland sheepdogs and West Highland White terriers. Data are presented as the mean \pm the standard error of the mean. UC, urothelial carcinoma. 


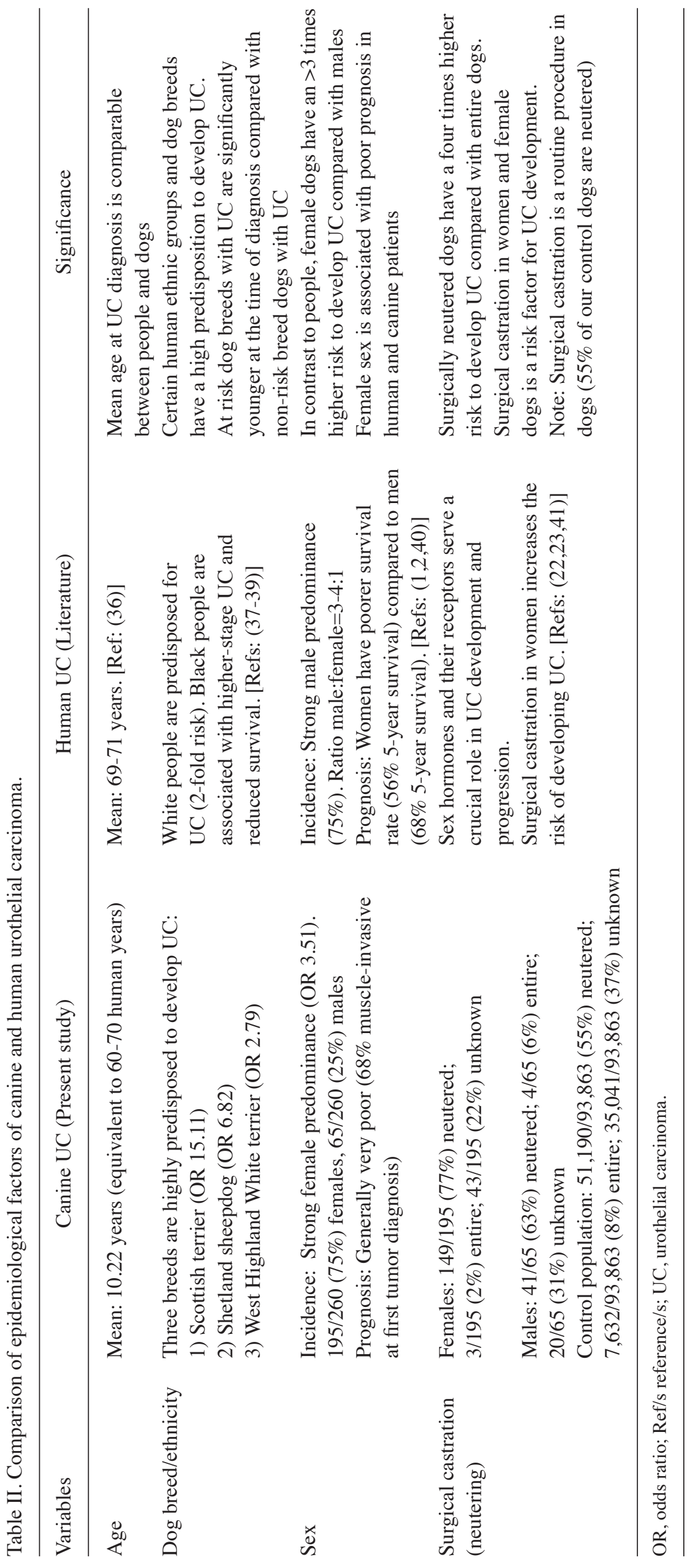



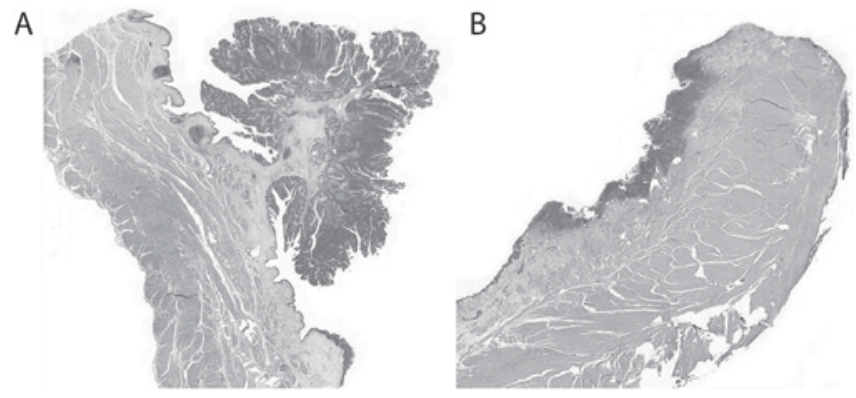

Figure 2. Microphotographs of urothelial carcinoma in the urinary bladder of two dogs with H\&E staining. Tumour growth was (A) papillary in a 6-year-old female entire giant schnauzer and (B) flat in an 11-year-old male neutered Cavalier King Charles spaniel, respectively. Magnification, (A) x3 and (B) $x 4$. H\&E, haematoxylin and eosin.
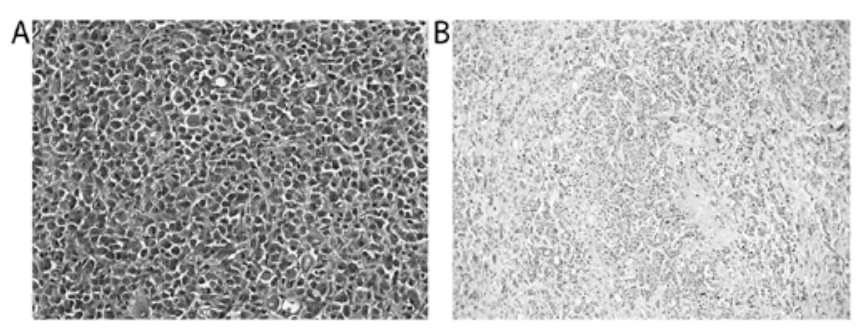

Figure 3. Microphotographs of two rare tumor variants in two dogs visualised using H\&E staining. (A) Primary urethral plasmacy toid urothelial carcinoma in a 9-year-old male neutered crossbreed dog. Round anaplastic cells grew in a sheet-like discohesive pattern. (B) Neuroendocrine carcinoma in the urinary bladder of a 13-year-old female neutered crossbreed dog. Highly anaplastic cells formed small cell nests and trabecules. Magnification, (A) x300 and (B) $x 100 . H \& E$, haematoxylin and eosin.

bladder UC cases. More than two thirds $(52 / 76,68 \%)$ were muscle-invasive tumors, which is compatible with high tumor stage. The tumor growth pattern of muscle-invasive UC could be assessed in 41/52 (79\%) cases, and it was more commonly flat $(30 / 41,73 \%)$ than papillary $(11 / 41,27 \%)$. This was in contrasts to the non-muscle-invasive UC, the vast majority of which $(22 / 24,92 \%)$ presented with a papillary growth, with only rare flat tumor growth $(2 / 24,8 \%)$. Non-papillary, flat tumor growth was statistically significantly strongly associated with tumor muscle invasion (OR $31.00,95 \%$ CI 66.24-153.95).

Conventional, not further classifiable infiltrating UC was the most common type of tumor $(234 / 260,90 \%)$, followed by UC with divergent differentiation $(15 / 260,6 \%)$ (squamous [8/260, 3\%), glandular $(5 / 260,2 \%)$ or both $(2 / 260,1 \%)]$, and squamous cell carcinoma $(5 / 260,2 \%)$. One case was identified to be a bladder adenocarcinoma (not otherwise specified). Two rare urinary tract tumors, including primary urethral plasmacytoid UC and neuroendocrine bladder tumor, have been identified in one dog each (Fig. 3). Eight of the 234 not further classifiable infiltrating UC cases presented with certain characteristic histological features within a low percentage (1 to 20\%) of the studied tissue section, including micropapillary and microcystic growth (Fig. 4), neuroendocrine differentiation as well as the presence of neoplastic cells with plasmacytoid, rhabdoid, signet-ring cell, lipid-rich or glycogen-rich appearance (Fig. 5). Since the divergent

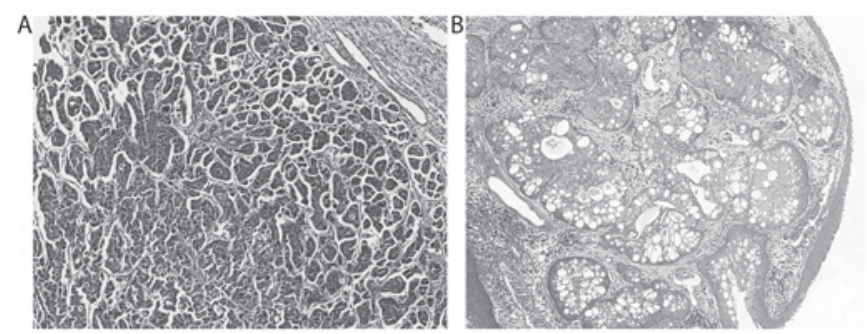

Figure 4. Microphotographs of urothelial carcinoma with different histological growth patterns in the urinary bladder of two dogs visualised using H\&E staining. (A) Micropapillary growth in a 10-year-old female neutered lurcher. (B) Microcystic growth in a 12-year-old male neutered miniature pinscher. Magnification, (A) x100 and (B) x50. H\&E, haematoxylin and eosin.

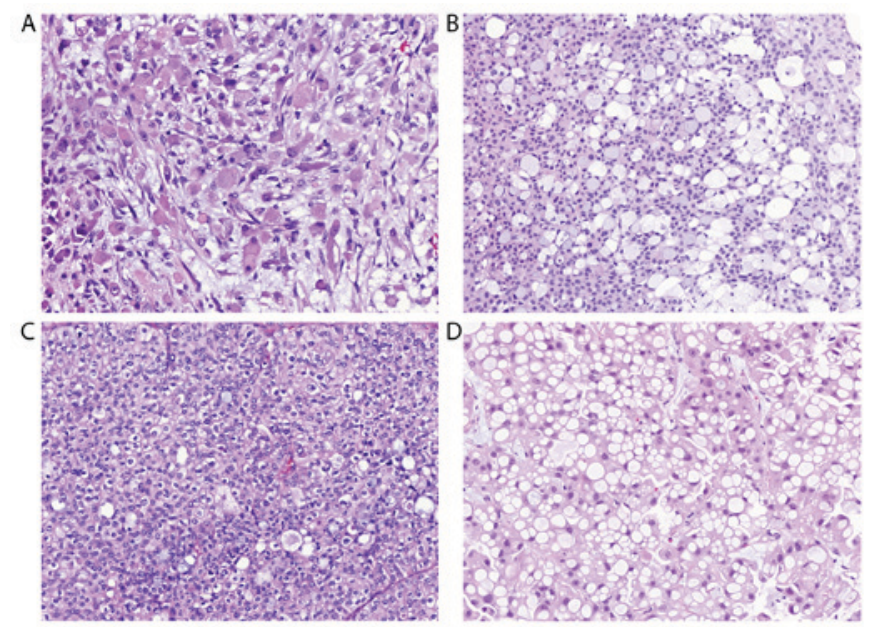

Figure 5. Microphotographs of urothelial carcinoma with different cellular features in the urinary bladder of four dogs visualised using H\&E staining. (A) Rhabdoid features in a 9-year-old female Labrador retriever. (B) Presence of signet ring cells in a 12-year-old female neutered Labrador retriever. (C) Glycogen rich areas in a 10-year-old male Jack Russell terrier. (D) Lipid rich areas in a 9-year-old female neutered West Highland White terrier. Magnification, (A) x300 and (B-D) x200. H\&E, haematoxylin and eosin.

differentiation was however only observed in a minor portion of the examined tumor tissue, these tumors were diagnosed as not further classifiable infiltrating UC and not as UC with divergent differentiation. The urethral plasmacytoid UC was found in a 9-year-old male neutered crossbreed dog with a history of dyschezia and prostate enlargement. The submitted and histologically examined tissue was limited to urethra. Prostate tissue was not available and no further information about the follow-up of this dog was given. The neuroendocrine bladder tumour was diagnosed in a 13-year-old female neutered $7 \mathrm{~kg}$ crossbreed dog with reported weight loss since a few months and a history of polyuria and polydipsia, pollakisuria, haematuria, and increasing lethargy over a period of less than 2 months. The tumor was diagnosed based on cystoscopy and biopsy. Due to late tumor diagnosis with very advanced disease and poor prognosis, the dog's owner decided against surgery and chemotherapy and elected euthanasia 3 weeks after the tumor diagnosis was made. Consent for a full post-mortem examination was given and confirmed the primary bladder tumor with metastases in lung, medial iliac and sacral lymph nodes. 


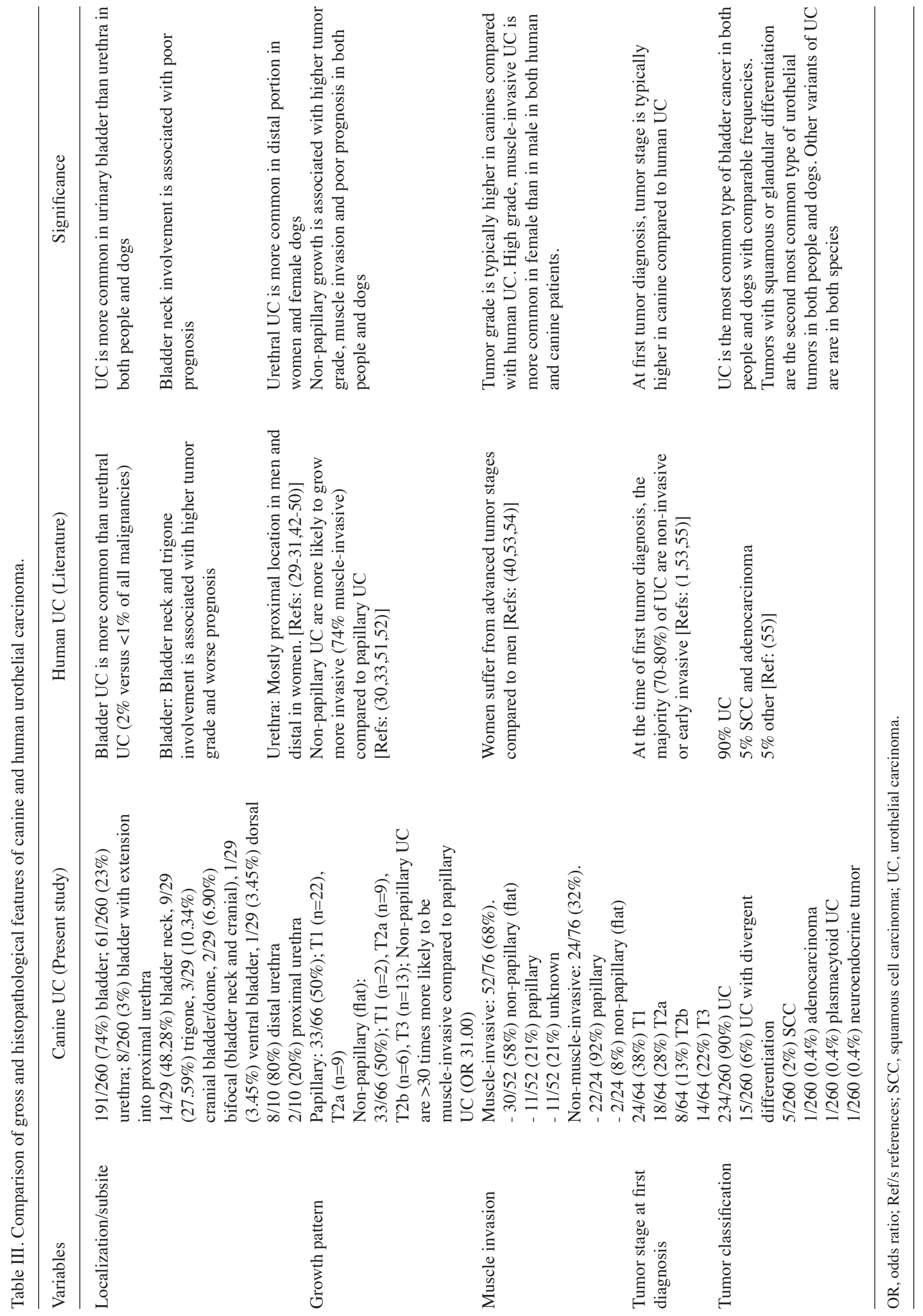


Finally, non-conventional UC was associated with muscle-invasive tumor growth $(\mathrm{P}<0.001)$, being compatible with higher tumor stage.

\section{Discussion}

The present study confirms the previously observed striking epidemiological and pathological similarities between canine and human UC $(6,14)$. The number of comparative human and canine bladder cancer studies available in the literature is still very low, with the most relevant studies being done in North America (15-20). The present study is the largest study with comparative epidemiology and histology, which includes 260 cases. It demonstrates that similar to the situation in people, canine UC mainly affects elderly patients. The mean age of dogs diagnosed with UC was over 10 years, which is equivalent to approximately 60 to 70 human years (21), compatible with the mean age of people diagnosed with UC. Both canine and human UC have a strong sex predilection. While men are at a three-fold higher risk to develop UC compared to women, the situation is exactly the opposite in dogs. Similar to the situation in people, the reason for the sex predisposition is unknown in dogs. Behavioral, anatomic, physiological and hormonal gender differences have been discussed, but remain speculative $(22,23)$. It is important to take into account that most of the dogs in the present study were surgically castrated which could potentially have contributed to the observed female predisposition for developing UC. Indeed, neutering appeared as a risk factor for UC for both female and male dogs in the present and previous studies (6). Interestingly, a similar phenomenon can be seen in women with a history of bilateral oophorectomy, who have a higher risk to develop UC $(22,23)$. Given that castration causes a change in the level of sex hormones and their receptors, these proteins likely play a major role in the carcinogenesis of UC. Previous studies have shown that reduced androgen receptor and increased estrogen receptor-beta expression are associated with higher tumor stage and grade, respectively (24-28).

Another remarkable feature of canine UC is its high predisposition for certain dog breeds $(14,17)$, which we could confirm in our study. Dogs of the same breed are genetically closely related and can be compared to related human families. The Scottish terrier has been previously shown to be the breed with the highest risk (up to over 20-fold) to develop UC $(14,17)$. Present results confirm this observation, which raises high suspicion of a strong genetic component of UC in certain dog breeds. Interestingly, the three dog breeds with highest risk to develop UC were significantly younger at the time of tumor diagnosis, compared to other dog breeds. The age difference was just over one year, which would be equivalent to approximately 7 human years (21).

Pathologically, canine and human UC are very similar, both grossly and histologically. UC affects most commonly the urinary bladder in both people and dogs and represents the most common (over 90\%) type of bladder cancer in both species. The lateral and posterior wall are the most common sites of UC in people (29-31), whereas dogs in our and previous studies tend to have UC within the neck and trigone area $(6,32)$. Reasons for the difference in preferred bladder subsites of UC between men and dogs are not known, but they may be due to the different orientation of the bladder within the body, leading to different intravesical urine flow. The prognostic significance of the different tumor bladder subsites is reported with some conflicting results, but involvement of neck or trigone appears to be associated with higher tumor stage and worse prognosis in people $(29,31)$. This could potentially explain the higher UC tumor stages in dogs compared to men.

The vast majority, between 80 and $90 \%$, of human UC grow papillary (30). Non-papillary (flat or endophytic) growth is much less common in people. This is in contrast to the findings of the here studied canine tumors which presented with an equal number of papillary and flat tumors. Interestingly, non-papillary growth has been demonstrated to be associated with higher tumor grade, muscle invasion and poor prognosis in people $(30,33)$. The studied canine data confirms this observation.

Histologically, the present study demonstrates that UC of people and dogs are strikingly similar and directly comparable. Non-conventional UC are less common in both species and most frequently comprise UC with divergent, glandular and/or squamous, differentiation. Other UC subtypes are rare in both species, but important to recognize given their association with poor prognosis (34). In the studied canine population, one case of primary urethral plasmacytoid UC in a male neutered dog, and one case of neuroendocrine tumor in the bladder of a female neutered dog were identified. Both tumors were high grade with evidence of intravascular neoplastic growth in the plasmacytoid UC and widespread lymph node and distant metastases in the neuroendocrine tumor, respectively. Neither of these two tumor types have been described in any non-experimental animal species and are herein reported for the first time as spontaneous tumors in an animal species. Other histological features, including micropapillary and microcystic growth, and rhabdoid, signet-ring cell, lipid-rich and glycogen-rich appearance of neoplastic cells were observed in a small proportion of the herein studied canine cases. These histological tumor features are poorly described in animals, but are well known features of UC in people $(13,34,35)$. These results therefore further support the value and suitability of the dog as animal model for UC in people.

In conclusion, canine and human UC are epidemiologically and pathologically directly comparable, which promotes the dog as a valuable animal model. Canine UC has an extraordinarily high predisposition for certain genetically closely related dog breeds which suggests a strong genetic component which needs further investigation. Female surgically neutered dogs are at highest risk to develop UC which leads to the speculation that sex hormones are crucial in the carcinogenesis of UC. Given the typically high tumor grades in dogs, canine UC appears to mimic the malignant muscle-invasive form of UC in people which is known to lack a well-established animal model that is so urgently needed to discover new therapeutic options. Finally, rare urinary tract tumors, including plasmacytoid UC and neuroendocrine tumor, occur spontaneously in dogs and are herein described for the first time in a non-experimental animal species. 


\section{Acknowledgements}

The authors would like to thank Mrs. Deepthi Chandy (Bridge Pathology Ltd., Bristol, UK) for her valuable assistance in producing high quality histological slides and Chris Nolan (City Hospital, University Nottingham, UK) for digitalizing all of the slides.

\section{Funding}

The present study was supported by PetPlan Charitable Trust (grant no. 542127), PetSavers and the University of Nottingham Interdisciplinary Centre for Analytical Science (UNICAS).

\section{Availability of data and materials}

The datasets used and/or analyzed during the current study are available from the corresponding author on reasonable request.

\section{Authors' contributions}

SdB, EW, TS and DG contributed to the acquisition and analysis of the epidemiological data. SdB, LGR, BDR and TS performed the histological examinations. SdB, EW and DG performed the statistical analysis. SdB, SAB, BDR and NPM compared the canine data with data available from scientific human literature. SdB and NPM were major contributors in writing the manuscript. All authors critically reviewed, read and approved the final manuscript.

\section{Ethics approval and consent to participate}

The present study was reviewed and approved by the University of Nottingham's Ethical Review Committee (Nottingham, UK). Informed written consent was obtained from the dog owners upon sample submission.

\section{Consent for publication}

Informed written consent was obtained from the dog owners upon sample submission.

\section{Competing interests}

The authors declare that they have no competing interests.

\section{References}

1. Ferlay J, Soerjomataram I, Dikshit R, Eser S, Mathers C, Rebelo M, Parkin DM, Forman D and Bray F: Cancer incidence and mortality worldwide: Sources, methods and major patterns in GLOBOCAN 2012. Int J Cancer 136: E359-E386, 2015.

2. Ferlay J, Soerjomataram I, Ervik M, Dikshit R, Eser S, Mathers C, Rebelo M, Parkin DM, Forman D and Bray F: GLOBOCAN 2012 v1.0, Cancer Incidence and Mortality Worldwide: IARC cancer base no. 11 [Internet]. Lyon, France: International Agency for Research on Cancer; 2013. Available from: http://globocan. iarc.fr, accessed on 04/12/2017.

3. John BA and Said N: Insights from animal models of bladder cancer: Recent advances, challenges, and opportunities Oncotarget 8: 57766-57781, 2017.

4. Kobayashi T, Owczarek TB, McKiernan JM and Abate-Shen C: Modelling bladder cancer in mice: Opportunities and challenges. Nat Rev Cancer 15: 42-54, 2015.
5. Ding J, Xu D, Pan C, Ye M, Kang J, Bai Q and Qi J: Current animal models of bladder cancer: Awareness of translatability (Review). Exp Ther Med 8: 691-699, 2014.

6. Knapp DW, Glickman NW, Denicola DB, Bonney PL, Lin TL and Glickman LT: Naturally-occurring canine transitional cell carcinoma of the urinary bladder A relevant model of human invasive bladder cancer. Urol Oncol 5: 47-59, 2000.

7. Choy K and Fidel J: Tolerability and tumor response of a novel low-dose palliative radiation therapy protocol in dogs with transitional cell carcinoma of the bladder and urethra. Vet Radiol Ultrasound 57: 341-351, 2016.

8. Knapp DW, Ruple-Czerniak A, Ramos-Vara JA, Naughton JF Fulkerson CM and Honkisz SI: A nonselective cyclooxygenase inhibitor enhances the activity of vinblastine in a naturally-occurring canine model of invasive urothelial carcinoma. Bladder Cancer 2: 241-250, 2016.

9. Arnold EJ, Childress MO, Fourez LM, Tan KM, Stewart JC Bonney PL and Knapp DW: Clinical trial of vinblastine in dogs with transitional cell carcinoma of the urinary bladder. J Vet Intern Med 25: 1385-1390, 2011.

10. Abbo AH, Jones DR, Masters AR, Stewart JC, Fourez L and Knapp DW: Phase I clinical trial and pharmacokinetics of intravesical mitomycin $\mathrm{C}$ in dogs with localized transitional cell carcinoma of the urinary bladder. J Vet Intern Med 24: 1124-1130, 2010.

11. Henry CJ, McCaw DL, Turnquist SE, Tyler JW, Bravo L, Sheafor S, Straw RC, Dernell WS, Madewell BR, Jorgensen L, et al: Clinical evaluation of mitoxantrone and piroxicam in a canine model of human invasive urinary bladder carcinoma. Clin Cancer Res 9: 906-911, 2003.

12. Moch H, Humphrey PA, Ulbright TM and Reuter VE (eds.): Tumours of the urinary tract. In: WHO Classification of Tumours of the Urinary System and Male Genital Organs (4th edition). IARC, Lyon: pp77-pp134, 2016.

13. Humphrey PA, Moch H, Cubilla AL, Ulbright TM and Reuter VE: The 2016 WHO Classification of Tumours of the Urinary System and Male Genital Organs-Part B: Prostate and bladder tumours. Eur Urol 70: 106-119, 2016.

14. Knapp DW, Ramos-Vara JA, Moore GE, Dhawan D, Bonney PL and Young KE: Urinary bladder cancer in dogs, a naturally occurring model for cancer biology and drug development. ILAR J 55: 100-118, 2014.

15. Valli VE, Norris A, Jacobs RM, Laing E, Withrow S, Macy D, Tomlinson J, McCaw D, Ogilvie GK, Pidgeon G, et al: Pathology of canine bladder and urethral cancer and correlation with tumour progression and survival. J Comp Pathol 113: 113-130, 1995.

16. Patrick DJ, Fitzgerald SD, Sesterhenn IA, Davis CJ and Kiupel M: Classification of canine urinary bladder urothelial tumours based on the World Health Organization/International Society of Urological Pathology consensus classification. J Comp Pathol 135: 190-199, 2006.

17. Norris AM, Laing EJ, Valli VE, Withrow SJ, Macy DW, Ogilvie GK, Tomlinson J, McCaw D, Pidgeon G and Jacobs RM: Canine bladder and urethral tumors: A retrospective study of 115 cases (1980-1985). J Vet Intern Med 6: 145-153, 1992.

18. Shapiro SG, Raghunath S, Williams C, Motsinger-Reif AA, Cullen JM, Liu T, Albertson D, Ruvolo M, Bergstrom Lucas A, Jin J, et al: Canine urothelial carcinoma: Genomically aberrant and comparatively relevant. Chromosome Res 23: 311-331, 2015.

19. Dhawan D, Paoloni M, Shukradas S, Choudhury DR, Craig BA, Ramos-Vara JA, Hahn N, Bonney PL, Khanna C and Knapp DW: Comparative gene expression analyses identify luminal and basal subtypes of canine invasive urothelial carcinoma that mimic patterns in human invasive bladder cancer. PLoS One 10: e0136688, 2015.

20. Decker B, Parker HG, Dhawan D, Kwon EM, Karlins E, Davis BW, Ramos-Vara JA, Bonney PL, McNiel EA, Knapp DW and Ostrander EA: Homologous mutation to human BRAF V600E is common in naturally occurring canine bladder cancer-evidence for a relevant model system and urine-based diagnostic test. Mol Cancer Res 13: 993-1002, 2015.

21. Patronek GJ, Waters DJ and Glickman LT: Comparative longevity of pet dogs and humans: Implications for gerontology research. J Gerontol A Biol Sci Med Sci 52: B171-B178, 1997.

22. Dietrich K, Demidenko E, Schned A, Zens MS, Heaney J and Karagas MR: Parity, early menopause and the incidence of bladder cancer in women: A case-control study and meta-analysis. Eur J Cancer 47: 592-599, 2011.

23. Prizment AE, Anderson KE, Harlow BL and Folsom AR: Reproductive risk factors for incident bladder cancer: Iowa Women's Health Study. Int J Cancer 120: 1093-1098, 2007. 
24. Kauffman EC, Robinson BD, Downes M, Marcinkiewicz K, Vourganti S, Scherr DS, Gudas LJ and Mongan NP: Estrogen receptor- $\beta$ expression and pharmacological targeting in bladder cancer. Oncol Rep 30: 131-138, 2013.

25. Miyamoto H, Yao JL, Chaux A, Zheng Y, Hsu I, Izumi K, Chang C, Messing EM, Netto GJ and Yeh S: Expression of androgen and oestrogen receptors and its prognostic significance in urothelial neoplasm of the urinary bladder. BJU Int 109: 1716-1726, 2012.

26. Tuygun C, Kankaya D, Imamoglu A, Sertcelik A, Zengin K, Oktay M and Sertcelik N: Sex-specific hormone receptors in urothelial carcinomas of the human urinary bladder: A comparative analysis of clinicopathological features and survival outcomes according to receptor expression. Urol Oncol 29: 43-51, 2011.

27. Shen SS, Smith CL, Hsieh JT, Yu J, Kim IY, Jian W, Sonpavde G, Ayala GE, Younes M and Lerner SP: Expression of estrogen receptors-alpha and -beta in bladder cancer cell lines and human bladder tumor tissue. Cancer 106: 2610-2616, 2006.

28. Boorjian S, Ugras S, Mongan NP, Gudas LJ, You X, Tickoo SK and Scherr DS: Androgen receptor expression is inversely correlated with pathologic tumor stage in bladder cancer. Urology 64 383-388, 2004.

29. Xiao GQ and Rashid H: Bladder neck urothelial carcinoma: A urinary bladder subsite carcinoma with distinct clinicopathology. Int J Surg Pathol 23: 517-523, 2015.

30. Sciarra A, De Matteis A, Mariotti G, Voria G, Lucera R and Di Silverio F: Histopathological aspects of transitional cell carcinoma of the bladder: Analysis of 20 years experience. Int J Urol 11: 467-475, 2004.

31. Stephenson WT, Holmes FF, Noble MJ and Gerald KB: Analysis of bladder carcinoma by subsite. Cystoscopic location may have prognostic value. Cancer 66: 1630-1635, 1990.

32. Fulkerson CM and Knapp DW: Management of transitional cell carcinoma of the urinary bladder in dogs: A review. Vet J 205: 217-225, 2015

33. Guo A, Liu A and Teng X: The pathology of urinary bladder lesions with an inverted growth pattern. Chin J Cancer Res 28: $107-121,2016$

34. Amin M: Histological variants of urothelial carcinoma: Diagnostic, therapeutic and prognostic implications. Mod Pathol 22 (22 Suppl 2): S96-S118, 2009

35. Zhai QJ, Black J, Ayala AG and Ro JY: Histologic variants of infiltrating urothelial carcinoma. Arch Pathol Lab Med 131: 1244-1256, 2007.

36. Madeb R and Messing EM: Gender, racial and age differences in bladder cancer incidence and mortality. Urol Oncol 22: 86-92, 2004

37. Yee DS, Ishill NM, Lowrance WT, Herr HW and Elkin EB Ethnic differences in bladder cancer survival. Urology 78: 544-549, 2011

38. Lee CT, Dunn RL, Williams C and Underwood W III: Racial disparity in bladder cancer: Trends in tumor presentation at diagnosis. J Urol 176: 927-933, 2006.

39. Hankey BF and Myers MH: Black/white differences in bladder cancer patient survival. J Chronic Dis 40: 65-73, 1987.

40. Tracey E, Watt H, Currow D, Young J and Armstrong B: Investigation of poorer bladder cancer survival in women in NSW, Australia: A data linkage study. BJU Int 113: 437-448, 2014.
41. McGrath M, Michaud DS and De Vivo I: Hormonal and reproductive factors and the risk of bladder cancer in women. Am J Epidemiol 163: 236-244, 2006.

42. Annan AC, Stevens KA and Osunkoya AO: Urothelial carcinoma involving the ureteral orifice: A clinicopathologic analysis of 93 cases. Hum Pathol 65: 101-106, 2017.

43. Sui W, RoyChoudhury A, Wenske S, Decastro GJ, McKiernan JM and Anderson CB: Outcomes and prognostic factors of primary urethral cancer. Urology 100: 180-186, 2017.

44. Zhou M, Netto GJ and Epstein JI: Uropathology: Elsevier Saunders 1st Edition, 2012

45. Dayyani F, Hoffman K, Eifel P, Guo C, Vikram R, Pagliaro LC and Pettaway C: Management of advanced primary urethral carcinomas. BJU Int 114: 25-31, 2014.

46. Gakis G, Witjes JA, Compérat E, Cowan NC, De Santis M, Lebret T, Ribal MJ and Sherif AM; European Association of Urology: EAU guidelines on primary urethral carcinoma. Eur Urol 64: 823-830, 2013

47. Svatek RS, Clinton TN, Wilson CA, Kamat AM, Grossman HB, Dinney CP and Shah JB: Intravesical tumor involvement of the trigone is associated with nodal metastasis in patients undergoing radical cystectomy. Urology 84: 1147-1151, 2014.

48. Kobayashi S, Fujii Y, Koga F, Yokoyama M, Ishioka J, Matsuoka Y, Numao N, Saito K, Masuda H and Kihara K: Impact of bladder neck involvement on progression in patients with primary non-muscle invasive bladder cancer: A prospective validation study. Urol Oncol 32: 38.e29-e36, 2014.

49. Reis LO, Ferreira F, Almeida M and Ferreira U: Urethral carcinoma: Critical view on contemporary consecutive series. Med Oncol 28: 1405-1410, 2011.

50. Swartz MA, Porter MP, Lin DW and Weiss NS: Incidence of primary urethral carcinoma in the United States. Urology 68: 1164-1168, 2006.

51. Terada T: Inverted variant of urothelial carcinoma of the urinary bladder: A report of three cases and a proposal for a new clinicopathologic entity. Int J Clin Ex Pathol 6: 766-770, 2013.

52. Kern WH: The grade and pathologic stage of bladder cancer. Cancer 53: 1185-1189, 1984

53. Babjuk M, Burger M, Zigeuner R, Shariat SF, van Rhijn BW, Comperat E, Sylvester RJ, Kaasinen E, Böhle A, Palou Redorta J, et al: EAU guidelines on non-muscle-invasive urothelial carcinoma of the bladder: Update 2013. Eur Urol 64: 639-653, 2013.

54. Mungan NA, Aben KK, Schoenberg MP, Visser O, Coebergh JW, Witjes JA and Kiemeney LA: Gender differences in stage-adjusted bladder cancer survival. Urology 55: 876-880, 2000.

55. Amin MB and Tickoo SK: Genitourinary diagnostic pathology. IInd Edition: Elsevier, 2016.

This work is licensed under a Creative Commons Attribution-NonCommercial-NoDerivatives 4.0 International (CC BY-NC-ND 4.0) License. 